University of Texas at El Paso

ScholarWorks@UTEP

$5-2020$

\title{
How to Estimate the Stiffness of the Multi-Layer Road Based on Properties of Layers: Symmetry-Based Explanation for Odemark's Equation
}

\author{
Edgar Daniel Rodriguez Velasquez \\ Universidad de Piura, edgar.rodriguez@udep.pe \\ Vladik Kreinovich \\ The University of Texas at El Paso, vladik@utep.edu \\ Olga Kosheleva \\ The University of Texas at El Paso, olgak@utep.edu \\ Nguyen Hoang Phuong \\ Thang Long University, nhphuong2008@gmail.com
}

Follow this and additional works at: https://scholarworks.utep.edu/cs_techrep

Part of the Applied Mathematics Commons

Comments:

Technical Report: UTEP-CS-20-49

\section{Recommended Citation}

Rodriguez Velasquez, Edgar Daniel; Kreinovich, Vladik; Kosheleva, Olga; and Phuong, Nguyen Hoang, "How to Estimate the Stiffness of the Multi-Layer Road Based on Properties of Layers: Symmetry-Based Explanation for Odemark's Equation" (2020). Departmental Technical Reports (CS). 1436.

https://scholarworks.utep.edu/cs_techrep/1436

This Article is brought to you for free and open access by the Computer Science at ScholarWorks@UTEP. It has been accepted for inclusion in Departmental Technical Reports (CS) by an authorized administrator of ScholarWorks@UTEP. For more information, please contact Iweber@utep.edu. 


\title{
How to Estimate the Stiffness of a Multi-Layer Road Based on Properties of Layers: Symmetry-Based Explanation for Odemark's Equation
}

\author{
Edgar Daniel Rodriguez Velasquez, Vladik Kreinovich, Olga Kosheleva, and \\ Hoang Phuong Nguyen
}

\begin{abstract}
When we design a road, we would like to check that the current design provides the pavement with sufficient stiffness to withstand traffic loads and climatic conditions. For this purpose, we need to estimate the stiffness of the road based on stiffness and thickness of its different layers. There exists a semi-empirical formula for this estimation. In this paper, we show that this formula can be explained by natural scale-invariance requirements.
\end{abstract}

\section{Formulation of the Problem}

Need to estimate stiffness of multi-layer roads. Most roads consist of several layers:

- First, there is a layer of soil - if needed, stabilized by adding lime, cement, etc.

- Then there is a layer - usually compacted - of crushed rocks.

- Finally, an asphalt or concrete layer is placed on top.

The road has to have a certain stiffness, i.e., a certain value of the modulus characterizing this stiffness. It is therefore desirable to estimate the stiffness of the designed

Edgar Daniel Rodriguez Velasquez

Department of Civil Engineering, Universidad de Piura in Peru (UDEP), Av. Ramón Mugica 131

Piura, Peru, e-mail: edgar.rodriguez@udep.pe

and

Department of Civil Engineering, University of Texas at El Paso, 500 W. University

El Paso, TX 79968, USA, e-mail: edrodriguezvelasquez@miners.utep.edu

Vladik Kreinovich and Olga Kosheleva

University of Texas at El Paso, El Paso, Texas 79968, USA

e-mail:vladik@utep.edu,olgak@utep.edu

Hoang Phuong Nguyen

Division Informatics, Math-Informatics Faculty, Thang Long University, Nghiem Xuan Yem Road Hoang Mai District, Hanoi, Vietnam, e-mail: nhphuong2008@gmail.com 
road with the layers of given thickness. In other words, we need to be able to solve the following problem:

- we know the modulus $E_{i}$ and the thickness $h_{i}$ of each layer;

- based on this information, we need to estimate the overall modulus $E$ of the road.

Odemark's equation. One of the methods for solving this problem was proposed in 1949 by N. Odemark [4]; his formula is

$$
E=\left(\frac{\sum_{i} h_{i} \cdot \sqrt[3]{E_{i}}}{\sum_{i} h_{i}}\right)^{3} .
$$

This formula is still in use; see, e.g., $[3,6]$.

Comment. The formula (3) corresponds to the usual case when different layers have similar Poisson ratios. For situations when the Poisson ratios of different layers are significantly different, there are more accurate versions of this formula.

How can we explain this formula. Odemark's formula is based on a simplified mechanical model of the pavement, where many important factors are ignored to make a simple formula possible. In principle, several different simplifications are possible; the formula produced by this particular simplification has been confirmed by empirical data. How can we explain this formula?

What we do in this paper. In this paper, we provide a theoretical explanation for this formula, an explanation based on the ideas of symmetry - namely, on the ideas of scale-invariance.

\section{Scale-Invariance: Reminder}

To measure a physical quantity, we need to select a measuring unit. In some cases, there is a physically natural unit - e.g., in the micro-world, we can use the electric charge of an electron as a natural measuring unit for electric charges. However, in many other situations, there is no such fixed unit. In such cases, it is reasonable to require that the dependence between the physical properties remains the same -i.e., described by the same formula - if we change the measuring unit.

If we replace the original measuring unit with a unit which is $\lambda$ times smaller, then all numerical values of the quantity will be multiplied by $\lambda: x \rightarrow \lambda \cdot x$. This transformation is known as re-scaling, and invariance with respect to this transformation is known as scale-invariance. Scale invariance is ubiquitous in physics; see, e.g., $[2,5]$. 


\section{Towards an Explanation}

Analysis of the problem. Let us first consider the simplified case when all the layers have the same thickness. The overall stiffness $E$ is the "average" stiffness, i.e., the stiffness that the road would have if all its layers have the same stiffness $E$.

Let us denote the overall effect of $n$ layers with stiffness $E_{1}, \ldots, E_{n}$ by

$$
E_{1} * \ldots * E_{n},
$$

for an appropriate combination operation $a * b$. In these terms, the stiffness of the $n$ layer road in which each layer has stiffness $E$ is described by the formula $E * \ldots * E$. Thus, the desired overall effect $E$ can be described by the formula

$$
E * \ldots * E=E_{1} * \ldots * E_{n} .
$$

The air layer with 0 stiffness does not contribute to the overall stiffness, so we should have $a * 0=a$.

If we have layers of different thickness $h_{i}$, then we can divide each of these layers into parts of the same thickness, and apply the same formula (2), i.e., we get

$$
\begin{gathered}
E * \ldots * E\left(h_{1}+\ldots+h_{n} \text { times }\right)= \\
E_{1} * \ldots * E_{1}\left(h_{1} \text { times }\right) * \ldots * E_{n} * \ldots * E_{n}\left(h_{n} \text { times }\right) .
\end{gathered}
$$

Natural properties of the combination operation $a * b$. In the first approximation, we can ignore the dependence on the order, and assume that $a * b=b * a$, i.e., assume that the combination operation is commutative.

It is also reasonable to assume that the result of applying this operation to a 3layer road does not depend on which layer we start with, i.e., that we should have $a * b * c=(a * b) * c=a *(b * c)$. In other words, the combination operation should be associative.

If we made one the layers stiffer, the stiffness of the road should increase. So, the combination operation should be strictly monotonic: if $a<a^{\prime}$, then

$$
a * b<a^{\prime} * b .
$$

Small changes in $E_{i}$ should lead to small changes in the overall stiffness. In mathematical terms, this means that the combination operation should be continuous.

Finally, we require that the combination operation be scale-invariant, i.e., that if $a * b=c$, then, for every $\lambda$, we should have the same relation for re-scaled values $\lambda \cdot a, \lambda \cdot b$, and $\lambda \cdot c$ :

$$
(\lambda \cdot a) *(\lambda \cdot b)=\lambda \cdot c
$$


Main result. We will show that every commutative, associative, strictly monotonic, continuous, and scale-invariant combination operation for which $a * 0=a$ has the form

$$
a * b=\left(a^{p}+b^{p}\right)^{1 / p}
$$

for some $p>0$.

Discussion. In other words, $a * b=c$ is equivalent to $a^{p}+b^{p}=c^{p}$, and, more generally, that $a * \ldots * b=c$ means that $a^{p}+\ldots+b^{p}=c^{p}$. In view of the formula (3), this means that

$$
\left(\sum_{i} h_{i}\right) \cdot E^{p}=\sum_{i} h_{i} \cdot E_{i}^{p},
$$

hence

$$
E=\left(\frac{\sum_{i} h_{i} \cdot E_{i}^{p}}{\sum_{i} h_{i}}\right)^{1 / p} .
$$

For $p=1 / 3$, we get exactly Odemark's formula!

\section{Proof of the Main Result}

$1^{\circ}$. Let us first prove that the operation $a * b$ has the form $f\left(f^{-1}(a)+f^{-1}(b)\right)$ for some monotonic function $f(a)$. In other words, we want to prove that $a * b=c$ is equivalent to

$$
f^{-1}(a)+f^{-1}(b)=f^{-1}(c),
$$

or, equivalently, that $f(a) * f(b)=f(c)$ is equivalent to $a+b=c$, i.e., that

$$
f(a+b)=f(a) * f(b) .
$$

Indeed, let us take $f(1) \stackrel{\text { def }}{=} 1$. Then, for every natural number $m$, we take

$$
f(m) \stackrel{\text { def }}{=} 1 * \ldots * 1(m \text { times }) .
$$

In this case indeed,

$$
\begin{gathered}
f(m) * f\left(m^{\prime}\right)=1 * \ldots * 1(m \text { times }) * 1 * \ldots * 1\left(m^{\prime} \text { times }\right)= \\
1 * \ldots * 1\left(m+m^{\prime} \text { times }\right)=f\left(m+m^{\prime}\right),
\end{gathered}
$$

i.e., we have the desired property (7).

Due to monotonicity, for each natural number $n$, we have we

$$
0 * \ldots * 0(n \text { times })=0<0 * \ldots * 0 * 1=1,
$$

and 


$$
1=0 * \ldots * 0 * 1<1 * \ldots * 1 \text { ( } n \text { times }) \text {. }
$$

From

$$
0 * \ldots * 0(n \text { times })<1<1 * \ldots * 1 \text { ( } n \text { times })
$$

and continuity of the combination operation, we conclude that there exists a value $v_{n}$ for which

$$
v_{n} * \ldots * v_{n}(n \text { times })=1 \text {. }
$$

We will then take

$$
f\left(\frac{1}{n}\right)=v_{n}
$$

We will then define

$$
f\left(\frac{m}{n}\right) \stackrel{\text { def }}{=} f\left(\frac{1}{n}\right) * \ldots * f\left(\frac{1}{n}\right)(m \text { times }) .
$$

One can check that for thus defined function $f(a)$, we indeed always have the formula (7) for rational values $a$ and $b$, and by continuity, we can extend the function $f(a)$ to all non-negative real values $a$.

$2^{\circ}$. Let us now prove that the inverse function $f^{-1}(a)$ is a power function - and thus, its inverse is also a power function.

Indeed, in terms of the formula (6), scale-invariance means that if the formula (6) is satisfied, then we have

$$
f^{-1}(\lambda \cdot a)+f^{-1}(\lambda \cdot b)=f^{-1}(\lambda \cdot c) .
$$

Let us denote $p \stackrel{\text { def }}{=} f^{-1}(a), q \stackrel{\text { def }}{=} f^{-1}(b), r \stackrel{\text { def }}{=} f^{-1}(c)$, so that $a=f(p), b=f(q)$, and $c=f(r)$. Let us also denote $t_{\lambda}(x) \stackrel{\text { def }}{=} f^{-1}(\lambda \cdot f(x))$, so that

$$
\begin{aligned}
& t_{\lambda}(p)=f^{-1}(\lambda \cdot f(p))=f^{-1}(\lambda \cdot a), \\
& t_{\lambda}(q)=f^{-1}(\lambda \cdot f(q))=f^{-1}(\lambda \cdot b),
\end{aligned}
$$

and

$$
t_{\lambda}(r)=f^{-1}(\lambda \cdot f(r))=f^{-1}(\lambda \cdot c) .
$$

In this form, scale-invariance takes the following form: if $p+q=r$, then $t_{\lambda}(p)+$ $t_{\lambda}(q)=t_{\lambda}(r)$. In other words, we have $t_{\lambda}(p+q)=t_{\lambda}(p)+t_{\lambda}(q)$ for all $p$ and $q$.

For integer values $p=n$, we thus have

$$
t_{\lambda}(1)=t_{\lambda}\left(\frac{1}{n}\right)+\ldots+t_{\lambda}\left(\frac{1}{n}\right)(n \text { times })=n \cdot t_{\lambda}\left(\frac{1}{n}\right),
$$

Thus

$$
t_{\lambda}\left(\frac{1}{n}\right)=\frac{1}{n} \cdot t_{\lambda}(1)
$$

Similarly, for evert $m$, we have 


$$
t_{\lambda}\left(\frac{m}{n}\right)=t_{\lambda}\left(\frac{1}{n}\right)+\ldots+t_{\lambda}\left(\frac{1}{n}\right)(m \text { times })=m \cdot t_{\lambda}\left(\frac{1}{n}\right)=\frac{m}{n} \cdot t_{\lambda}(1) .
$$

In other words, we conclude that

$$
t_{\lambda}(x)=x \cdot t_{\lambda}(1)
$$

for all rational $x$. By continuity, we can conclude that this property holds for all real values as well.

By definition of $t_{\lambda}(x)$, the equality (10) means that $f^{-1}(\lambda \cdot f(x))=t_{\lambda}(1) \cdot x$, i.e., that for $y=f(x)$, for which $x=f^{-1}(y)$, we have

$$
f^{-1}(\lambda \cdot y)=t_{\lambda}(1) \cdot f^{-1}(y) .
$$

It is known (see, e.g., [1]) that every continuous solution to this functional equation has the form $f^{-1}(x)=A \cdot x^{a}$ for some $A$ and $a$. Thus, we get the desired formula for the combination operation $a * b=f\left(f^{-1}(a)+f^{-1}(b)\right)$.

The result is proven.

Comment. The result from [1] can be easily proven, if instead of continuity, we make a stronger assumption that the combination operation - and thus, the function $f(a)$ - is differentiable. Indeed, in this case, $t_{\lambda}(1)$ is a differentiable function of $\lambda$, as a ratio of two differentiable functions. Thus, we can differentiate both sides of the equality (11) by $\lambda$ and take $\lambda=1$; then, we get

$$
x \cdot F^{\prime}(x)=c \cdot F
$$

where $F(x) \stackrel{\text { def }}{=} f^{-1}(x), F^{\prime}(x)$ means the derivative, and $c$ is the derivative of the expression $t_{\lambda}(1)$ when $\lambda=1$. The formula (12) can be rewritten as

$$
x \cdot \frac{d F}{d x}=c \cdot F
$$

i.e., equivalently,

$$
\frac{d F}{F}=c \cdot \frac{d x}{x}
$$

Integrating both parts, we get $\ln (F)=c \cdot \ln (x)+C$, where $C$ is the integration constant. Applying $\exp (z)$ to both sides, we get the desired power law.

\section{Acknowledgments}

This work was supported in part by the National Science Foundation grants 1623190 (A Model of Change for Preparing a New Generation for Professional Practice in Computer Science) and HRD-1242122 (Cyber-ShARE Center of Excellence). 


\section{References}

1. J. Aczel and J. Dhombres, Functional Equations in Several Variables, Cambridge University Press, Cambridge, UK, 1989.

2. R. Feynman, R. Leighton, and M. Sands, The Feynman Lectures on Physics, Addison Wesley, Boston, Massachusetts, 2005.

3. S. D. Guzzarlapudi, V. K. Adigopula, and R. Kumar, "Comparative study of flexible pavement layers moduli backcalculation using approximate and static approach", In: B. S. Thomas, R. Vyas, P. K. Goswami, L. J. Cseteny (eds.), Proceedings of the International Conference on Recent Trends in Engineering and Material Sciences (ICEMS'2016), Jaipur, India, March 17-19, 2016, Published in Material Today Proceedings, 2017, Vol. 4, No. 9, pp. 9812-9816.

4. N. Odemark, Investigations as to the Elastic Properties of Soils and Design of Pavements According to the Theory of Elasticity, Staten Vaeginstitut, Stockholm, 1949.

5. K. S. Thorne and R. D. Blandford, Modern Classical Physics: Optics, Fluids, Plasmas, Elasticity, Relativity, and Statistical Physics, Princeton University Press, Princeton, New Jersey, 2017.

6. US Federal Highway Administration (FHWA), Mechanistic Evaluation of Test Data from LTTP Flexible Pavement Test Sections, Vol. 1, Publication FHWA-RD-98-012, April 1998. 